Voix et Images

voixetimages

\title{
Dans les chances de la poésie
}

\section{Paul Chanel Malenfant}

Volume 16, numéro 1 (46), automne 1990

Les correspondants littéraires d'Alfred DesRochers

URI : https://id.erudit.org/iderudit/200889ar

DOI : https://doi.org/10.7202/200889ar

Aller au sommaire du numéro

\section{Éditeur(s)}

Université du Québec à Montréal

\section{ISSN}

0318-9201 (imprimé)

1705-933X (numérique)

Découvrir la revue

\section{Citer cet article}

Malenfant, P. C. (1990). Dans les chances de la poésie. Voix et Images, 16(1),

176-192. https://doi.org/10.7202/200889ar d'utilisation que vous pouvez consulter en ligne.

https://apropos.erudit.org/fr/usagers/politique-dutilisation/ 


\section{Poésie}

\section{Dans les chances de la poésie}

\section{par Paul Chanel Malenfant, Université du Québec à Rimouski}

Une année de congé sabbatique en France en compagnie d'œuvres admirables, entres autres celles de Guillevic, Leiris, Sacré, Réda, Hocquard, Viton, Gleize, Deguy, Albiach, Tellermann... me ramène en terrain plus familier, à l'enthousiasme de l'actuelle poésie d'ici. Est-ce l'euphorie des retrouvailles, l'effet bénéfique de la distance franchie, le bonheur occasionnel d'une saison faste de poèmes, certain chauvinisme ou telle indulgente myopie? Toujours est-il que les recueils posés sur ma table et destinés, en cette chronique pour moi inaugurale, aux risques comme aux doutes, aux humeurs comme aux déraisonnables émerveillements du commentaire critique, me paraissent porteurs, pour la plupart, de cette énergie et de cette vitalité sans compromis, de cette ferme exigence à l'égard des possibles du langage qui sont sans doute des traits marqués de la poésie québécoise telle qu'elle se pratique en cette "certaine fin de siècle».

Dès lors, la lecture relève du défi porté à sa propre subjectivité, comme si de la fascination à la complaisance... D'autant plus que j'arrive sur ce territoire, d'une part avec toute l'admiration que je destine à mon prédécesseur, André Brochu, dont je tiens pour inégalables la juste et perçutante "visée critique", l'éthique de lecture sans défaillance et la compétence passionnée de l'«art poétique"; d'autre part avec la voix voilée du trac, à la pensée des leçons de lecture d'un maître dont je crains de n'avoir pas toujours bien assimilé et le goût et la science. Que les livres, tels qu'en eux-mêmes, me viennent en aide. 
Si j'avais donc à définir d'emblée la correspondance que je souhaite établir entre les livres de poésie et ce discours, discours de l'«autre", susceptible de les donner un peu mieux à voir et à entendre, je retiendrais, à titre d'indicatifs critiques, ces titres du dernier recueil de Michael Delisle, Chose vocale ${ }^{1}$ : "L'émotion de l'autre ", "Mon frère en tête ", "Chose vocale", "Finir clair", "Rien de plus que ma voix dans le noir", "Une fois mis devant le choix", "Éblouis" et "La voix inaltérée». Tant il est vrai, me semble-t-il - et pour fonder en métaphore méthodologique cette nomenclature titulaire -, que la lecture "critique" du poème ne peut se situer que sous le coup de l'émotion (cette émotion de la forme, bien sûr...), qu'elle tient de l'élucidation claire-obscure mais fraternelle et altruiste, qu'elle est bien, [U]ne fois mis[e] devant le choix, voix portée et immédiatement perdue par la poésie même, à la fois liberté aveugle et ligne du risque.

"Chose vocale", en sorte: ce discours où je ne proposerai [r]ien de plus que ma voix dans le noir, en cela fidèle à la conscience que tout commentaire du poème n'est, selon la belle expression de Yves Bonnefoy, que savoir passionnel, savoir de la mémoire et du corps, savoir sans connaissance.

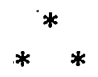

Tension de l'immédiateté des gestes quotidiens, de l'émotion décrite en son commencement, ce livre de Michael Delisle se place sous le signe de la surprise et du surgissement: Front qui colle au creux du dos, au bas du dos. Là, où l'épice commence. (p. 6) Les poses amoureuses,' les scènes de séduction, les stratégies de charme sont données ici comme autant de fêtes acoustiques du langage, autant d'occasions de porter la voix du poème: Pour moi, le poème était une façon d'entendre les choses. (p. 24) Cette proximité sensuelle avec la substance sémantique en son état le plus brut (Je passe mon doigt sur le mot. [p. 55]) et comme avant que le sens n'y advienne, conduit à une sorte de rêverie élémentaire de la salive, cet état amniotique de la langue. Par alchimie mimologique, les mots mêmes, alors, deviennent d'audacieuses choses. Ainsi, cette merveilleuse strophe, digne des affabulations sonores d'un Michel Leiris, où à la fois résonne la comptine et où s'inscrit, visuelle leçon des choses de la vie et de la mort, la bulle dessinée:

Alors, je pense quie les mots sont des bulles.

Je pense que ces bulles sont molles.

Que souvent, elles forment des globes.

Que souvent, au-dessus de petits lions enjoués,

Elles frissonnent devant la mort. (p. 44) 
Ce caractère ludique de la "voix" de Delisle ne se départit jamais d'une tranquille gravité: J'ai été cet homme-à-voix. / J'ai été cette encre noire, / Cassée au poème. (p. 48) Écrire le poème, c'est ici se tenir au plus près du souffle, de sa fluidité, de ses imprévisibles ruptures. Écrire comme on respire, donc, disponible à l'essentiel, au sens substantif, dans la paisible jubilation: Mais, écrire des poèmes sera toujours / Une joie sans comparaison. / Écrire des poèmes ou / Marcher dans le gravier sera toujours / Le lieu d'une sérénité. (p. 57).

Le procès figuratif ou mimétique de la voix de Delisle trouve une tout autre expression dans le grand poème de Jean-Paul Daoust, les Cendres bleues ${ }^{2}$. À l'ubiquité entre les mots et les choses, au dépouillement de la "chose vocale ", là où le poème - qu'il fréquente par stratégie le monde des images - n'a d'espace réel que sa voix. (p. 74), Daoust va préférer l'ample incantation narrative (comparable, en intensité, à celle mise en œuvre dans le grand livre de Fernand Ouellette, les Heures ${ }^{3}$ ), il va s'adonner à l'efflorescence débridée et volubile des images. Et tout se passe comme si Daoust répondait à l'inquiète interrogation de Delisle: Diras-tu quelque chose d'important sur le deuil? (p. 32) En effet, récit d'un homme blessé / D'avoir connu le sexe enfant / Six ans et demi (p. 7), lamento d'un enfant violé (ibid.), le texte de Daoust réalise admirablement, par la remontée vers l'enfance et par la découverte du sexe, tout ce travail de la mélancolie et du deuil amoureux.

À travers une syntaxe haletante qui se déploie tour à tour dans le lyrisme insistant du leitmotiv (Il m'aimait / je l'aimais) ou dans le rythme de la saccade, le poète se remémore les transes, les malédictions heureuses, les affres d'un premier amour. Flambant et neuf. Inoubliable alors, cette embellie amoureuse, cette figure de l'amant bleu que l'on retrouvera la tête enfouie dans des cendres bleues... et étoilees: Il avait des yeux bleus de dimanche de deuil. (p. 19) On connaît déjà, par d'autres textes, l'extravagance éclatée et tonique, une certaine phosphorescence de l'idiolecte Daoust. En cette histoire d'amour et de mort, animée d'un grand vertige igné que disent les images récurrentes du soleil couchant, des étoiles, des coups de haches et d'éclat parmi les volées de bois dans le " hangar" (lieu privilégié des rencontres sexuelles... où s'entendent, sous le mode consonantique, les halètements de jouissance du beau gars de vingt ans...), le récitant atteint cette fois au pathos polyphonique d'un chœur de tragédie.

Et Daoust excelle encore à traduire par images, façon Apollinaire peut-être, la troublante ambiguité de telle image pieuse: 
Mais de ces amants il $y$ en avait un

Aux yeux de harpe

Il me rappelait l'ange derrière l'autel

De l'église Notre-Dame-de-Bellerive (p. 7)

il discerne dans la lettre même des vocables, ces cruautés, ces intrigues, ces petites violences enfantines de la passion:

Et déjà j'apprenais à faire souffrir

À l'âge de l'alphabet

Des chenilles des papillons des fourmis

Des sauterelles donne-du-miel ou je te tue (p. 15)

enfin, il sait rendre à merveille la patine du souvenir, ce clair ravissement de la mémoire retrouvée:

Je sais qu'il y avait des jours où j'entrais

À la maison du Jardin de l'Enfance

Plus brillant plus lustré

Qu'un lit de cuivre fraîchement poli (p. 20)

Ces saillies mnémotechniques émues, assorties d'úne attention) discrète au grain du texte (ce "lui ", l'amant qui dissémine ses caresses sonores en la chaîne allitérative Plus brillant plus lustré / $Q u$ 'un lit de cuivre fraîchement poli), trouvent leur accomplissement dans la reconnaissance spéculaire, où l'écriture, soudain, ne se démêle plus de ce qu'elle narre:

Pourtant j'avais des doigts écrevisses

Et on écrivait

Toujours sur nos bûches de bois

Nos initiales (p. 22)

De ces écrevisses à ces écritures s'inscrit toute la méţamorphose qui conduit du réel à un texte, initiatique et initial, où il s'agit de dire (de réciter) - "toute la vérité rien que la vérité" - pour s'insurger contre l'interdit, pour dénier la durable étroitesse du "ce que les autres vont dire"... de "ça»! En guise de postface, une lettre solidaire de Paul Chamberland ajoute au poids de témoignage de ce livre déjà intense et très beau.

Micro-textes ${ }^{4}$, de Paul Rousseau, méritait cette année le prix Octave-Crémazie. Les coups d'envoi en poésie appellent une oreille empathique, disponible; en ce sens, j'ai parfois regretté que nombre des récipiendaires de ce prix, qui couronne un manuscrit de talent, restent confinés - petite gloire! - à leur premier livire. Malgré le clin d'œil "accrocheur-moderniste" d'un titre peu fonctionnel en regard du propos thématique du livre, ces Micro-textes (textes 
miniatures, mais encore, bien prosaïquement cette fois, "choses vocales" que ces "micros "...) offrent toutes les ressources et tous les plaisirs d'une manière de polar poétique: une jeune fille posée / avec son blond au plafond / [...] / un revolver qui rutile / bijou d'acier dans sa paume / menace de fer sur la nuque du chauffeur (p. 7-8). Ces fictions urbaines, construites selon les règles du suspense et où pullulent les objets symboliques de taille, engagent une fébrile (et combien virile!) dynamique de l'action, du péril et de l'exotisme géographique.

Souvent hyper-réaliste, la poésie parodie la consommation, la modernité gadget, l'américanité publicitaire, et elle atteint dans ses meilleurs moments, par effet de grand angle; à la surprise de l'abstraction:

\section{L'ouest brun des bottes de Cowboy}

enfoncé de gestes de pick-up Ford

trace des plaines où siffle du blé

les charolais ont soif (p. 10)

En d'autres occasions, le texte mime la sottise même de ce qu'il prétend dénoncer: Périr de bonheur détergent / pour une speakerine bien lavée / aux sourires ultra-brite / aux désirs encore plus blancs / l'aventure savonneuse (p. 11).

Ces écarts, dans la perspective photographique, relèvent sans doute de l'apprentissage du métier de reporter. Il faut cependant reconnaître à Paul Rousseau son'sens de l'image sensationnaliste du visage tranché de rouge aux lèvres (p.13) au mustang monté / pur sang (p. 20) - allié à une vision souvent baroque, "impure" et joyeusement moderne, de l'éclectisme du quotidien et du monde. Reste à émonder les scories, à moins que l'univers, ici décrit, réclame l'encombrement même de ses détritus: cette lassante contamination linguistique: du TV-Dinner (p. 11) au Bus Stop (p. 25); ces appositions systématiques qui créent effet de cumul et de saturation: du sein martyr (p. 13) à la souffrance pin-up (p. 26) jusqu'aux ciels doo bee doo (p. 29)! Vraiment, dans la prolifération désordonnée des objets du monde, les choses sont d'autres choses...

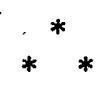

Si besoin était d'encourager Paul Rousseau en ce lieu du matérialisme exacerbé et ironique - façon Andy Warhol - où la nomination comme le dénombrement désignent, jusqu'à l'absurde, l'impossible exhaustivité du réel, il faudrait porter à son attention les derniers livres de Denise Boucher et de Denis Vanier, Paris polaroïd ${ }^{5}$ et les Stars du rodéo ${ }^{6}$. En effet, ces deux poètes sont passés maîtres dans l'art de la photographie sur le vif du sujet, du regard 
incisif sur l'insoutenable - admirable, aussi - crudité du monde. Ainsi, pour Denise Boucher, la vie est choquante de réalité (p. 29) tandis que chez Denis Vanier Le réel [est] délinquant (p. 43). Chez celui-ci, le poème procédera donc à une effraction langagière passionnée des scènes et des faits intolérables; chez celle-là, il énoncera cette compassion à la fois critique et attendrie, comme une tendresse mêlée de "spleen", à l'égard des assauts du réel.

Ainsi, l'itinéraire parisien de Denise Boucher s'ouvre sur une scène méditative: je me suis assise dans des jardins royaux / dessous la fenêtre de l'immense Colette / je me suis recueillie et j'ai pensé à toi / je t'en passe un papier mon ami de parcours (p. 13). Sur cet air mironien de «marche à l'amour», s'engage dans Paris une errance qui conduit la poète aux lieux les plus célèbres (le Palais Royal, Montmartre, NotreDame, le Luxembourg, la Seine, etc.) jusqu'à ces poèmes toponymiques que sont les rues des Blancs-Manteaux (là où devant un café crème vous m'avez offert Paris polaroïd: merci Denise!), du Cherche-Midi, Gît-le-Cœur, Pas-de-Mule... Bien sûr, il ne s'agira pas ici de la célébration guimauve d'un Paris rengaine mais bien, dans le mouvement de l'avancée et le dépaysement de l'ailleurs, d'une prise de conscience, cruelle et lucide à la fois, des sens (et des non-sens) de l'existence transitoire, de la fugacité des corps qui passent, du délire du monde, du manque à gagner de la poésie: même au prix de mes os j’ai des fins de mois difficiles (p. .41). En ce contexte, l'observation de.Boucher est cynique devant une meute de Japonais de fin d'été [...] leur âmé est dans leur caméra qui se précipitent sur tous les bonbons de chez Fauchon tandis que ce soir le poète Bonnefoy / donne ses mots en personne (p. 28). Tant il est vrai que la poésie qui consume ne se consomme pas, que l'achat des choses dépasse en pouvoir d'envoutement le don des mots... On rêverait alors à cette écriture du délire / la rumeur commerciale / le haut lieu du poème (p. 21).

Mais je colle à la terre / ne m'en délivrez pas (p. 22) réclame le poète après le constat irréversible: avant qu'un jour de fin je débarque de tout. / j'aurai senti sous l'aile les vents fous de la vie (p. 14). On pourrait entendre un apitoiement laconique en ces énoncés si ce n'était que le mal (et la passion) de vivre s'accompagnent, chez Denise Boucher, d'une perception aiguë, manifeste dans la langue même des poèmes, des contrastes et des chocs qui confèrent au réel son saisissant relief. La tendresse alors s'allie à l'ascèse ironique : car savoir laisser son cœur au congélateur / avec les restes glacés et les surgelés (p. 14); exotique, le désir tient de l'ordre du franc parler et de l'appétit vorace: je me taperai un de ces tarzan / je mettrai ma jupe en serpent / un beau marle descendu de sa côte d'ivoire (p. 53).

Ces divers registres, caractéristiques de la voix de Boucher, trouvent leur unité dans ce constant dessaisissement de soi en faveur de l'autre et de sa différence. Délibérément "mixte", le langage 
s'offre dans ses grandeurs comme dans ses misères; le trivial y soutient le pathétique et le pathos s'amenuise dans une écriture de la malédiction tranquille (bien près du bonheur, parfois..), de la chanson simple qui rend supportable la solitude: quand on est bien tout fin seul / que personne ne nous attend / qu'on tombe dans la Seine / sur un tableau de Leduc / vu par un dimanche d'avant (p. 26). La mélancolie de la musique allège les misères du corps et du cœur. Ainsi, en ces vers parfaits, où le malheur est tout entier porté par le bonheur de l'expression:

la misère a parfois des parfums de giroflée odorante

des lilas fleurissent à'même ma perte

je suis tombée bas dans cette peine de corps (p. 59)

J'aime encore, chez Denise Boucher, l'autonomie de telle phrase tirée de la vérité nue: j'entends bien mourir en toute liberté / de vieillesse et en amour (p. 47). L'extrême lisibilité rejoint ici la transparence de la sentence, de la confidence sans détour. Je suis aussi sollicité par cette conscience qui s'ouvre sur l'actualité du monde: $j$ 'ai vu aussi tomber le mur / le mur de rage de Berlin (p. 23); qui désigne, dans la naïveté du timide respect, tel lieu de classe interdit: la peur et le respect / bloquent mes désirs / devant le Waldorf Astoria (p. 40). Il est des lieux, donc, où l'on n'entre pas avec son "polaroïd"!

Paris polaroïd: album de voyage qui donne à voir, par delà les clichés des lieux; les âmes et les corps qui les habitent. Carnet de route, journal intime et voyage intérieur qui s'accomplit dans l'envergure de l'espace, dans la courageuse habitation d'un dehors: j'aimais mieux ne pas avoir de maison (p. 25). Car les voyages et la marche sont vécus comme départs perpétuels, choix de nomade pour la vie inchoative: j'emporterai ma vie sur un dos droit / prise du cœur par les pieds je partirai (p. 55).

Ces effets de tendresse qui envahissent le dernier livre de Denise Boucher, sans que le poète cesse pour autant, en un langage souvent furieux, voire efficacement chaotique, de broyer les horreurs du monde, je les retrace, en d'autres termes, dans les Stars du rodéo de Denis Vanier. Bien sûr, on trouve toujours chez ce poète la diction ravageuse, le goût pour l'imprécation délinquante et la vocifération blasphématoire: après la castration des sorciers / aux langues poreuses comme des chats / du virus de Jérusalem / aussi faut-il utiliser l'algue décapante / pour enlever ses hosties. (p. 14) Sans ces éclats fantasmatiques, sans la santé un peu perverse de cette colère, sans cette effraction des scènes somnolentes sous les rituels, nous n'aurions pas accès à la force de l'imaginaire perturbateur de Vanier.

En ce dernier livre, cependant, tels traits - jusque-là inédits, me semble-t-il? - du scénario familial viennent inférer à la poésie comme à la "délinquance du réel", une nouvelle solidarité avec le 
monde et les êtres. On entend la mère: mais faut-il s'envahir / de l'odeur de l'être aimé / c'est ce que ma mère disait / qu'il faut oublier pour toujours / le thé et les rennes et la colachine (p. 15). On porte compassion à l'égard des gestes de femmes: comme si leurs mères n'étaient pas des femmes. / mais celles qui lavent les mouchoirs du cœur / saignant (p. 33). On partage avec le père l'objet symbolique et la suite du monde: remercie ton père pour la canne / qui te permet d'arpenter / parmi les loups, la neige et les perles du nord (p. 26). Le poète inscrit encore de ces scènes sensibles, écologiques, et comme filtrées par une subtile synesthésie: j’ai les pieds bruns / comme une paysanne qui ne partage l'oie / que par ennui d'amandes / et de baumes de tofu (p. 21). Devant de telles réussites, sans doute faut-il croire à une nouvelle sagesse plutôt qu'à un morne assagissement.

Car la poésie de Vanier n'a rien perdu de sa force corrosive, de sa théâtralisation imaginaire, de son goût, que l'on pourrait comparer aux épopées rituelles de Mishima, pour l'horreur somptuaire: les filles sont belles comme le terrorisme / surtout quand elle s'ouvre / dans les glaieuls orange de la mort. (p. 37) Mais par l'inclusion parfois attendrie du référent familial, par de neuves allusions aux saveurs et aux substances singulières, le texte de Vanier gagne en séduction; il porte une sollicitation plus chaleureuse à l'égard du lecteur sans perdre ses forces de révulsion et de remise en cause. En ce contexte, l'efficacité de leur effet destroy et réflexif pourrait bien s'en trouver accrue.

Du point de vue de la forme, ce recueil présente des poèmes plus resserrés sur eux-mêmes comme pour une consolidation des éclats de voix. De très beaux vers basculent tout à coup dans le référent prosaïque, ce qui, chez Vanier, leur confère toujours un surcroît de poéticité: ses cheveux noirs écrasent la pulpe des pivoines / dans la fenêtre de la pizzeria / où s'ouvrent les jambes de la nuit (p. 47). De cet impeccable alexandrin on observera encore la diffusion harmonique des vocables bisyllabiques jusqu'à l'amenuisement des monosyllabes: il aérait sa poudre avec des gants de soie (p. 51). Je note encore, hachuré, texture sonore et sémantique drue, cet admirable tableau de la douleur dite:

le blessent au-dessus des hanches

son dos ploie sous la tremblée des feuilles

et son échafaud de nattes

pris dans les tailles d'acier

n'offre sans larmes ni gémissements

que les coloris amers de l'agonie (p. 67)

Un saisissant poème "La maison de l'horreur " (qu'il faut entendre comme l'horreur d'un centre hospitalier) clôt ce recueil de Vanier. Admirable texte de dénonciation, de patience et de lucidité. Je n'en 
cite qu'un trait, pour la beauté du cynisme et du désarroi: un bouton creux / où appuyer en cas d'urgence / pour noùs rappeler que l'intimité / est la propriété d'autrui (p. 73).

Avec les Stars du rodéo, on se plaît à croire que la nécessaire délinquance, celle qui refuse toute forme de récupération, peut, de connivence, s'entretenir avec la maturité du poème.

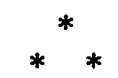

À l'instar de celui de Paul Rousseau, le livre de Louis Cornellièr, Neurones fragmentés ${ }^{7}$, constitue un premier recueil et il a mérité une mention au même prix Octave-Crémazie. Ici encore - fixation de ma part - le titre me gêne; ces "neurones fragmentés" ne me donnent à voir qu'un brutal essaimage de cellules en perte de pensée... Et le texte alors, comme expansion métaphorique de son titre?

"Scènes" et "Propositions", ces deux suites successives sont encadrées d'un "Prologue " et d'un "Épilogue»; telle se désigne, assez abstraitement, la structure du recueil. En l'espace d'une "chambre", lieu privilégié de l'intimité et de la clôture réflexive, où le mystère s'amorce et où le matin tarde à venir (p. 11), un homme va tenter d'explorer les différentes façades du non-dit (p. 13). Alors, fidèle à la consigne titulaire, Il]a pensée vole en éclats aux quatre coins de la chambre. Aux quatre coins du monde. (p. 12) D'emblée, s'avoue un cogito porté par la dissolution, le fragmentaire, la dissémination explosive. Car la quête de l'«origine" doit s'effectuer par delà le "sens " accablant, la vacuité permanente du sens (p. 13). Jusqu'ici, la portée existentielle du propos, pour n'être pas inédite, peut toujours retenir l'attention.

Mais bientôt, cet homme frictioñne la fiction (p. 13). Le charme philosophique est rompu. Nous assistons alors à un insistant massage rimal où la bruyante panoplie des lourds adverbes semble agir a contrario de toute détente poétique.

Un enfant griffe amoureusement une stèle dans un mouvement qui s'apparente étrangement à celui de l'étreinte sexuelle. Au bout d'un certain temps, un lustre profus émerge $d u$ mouvement. Le corps de l'enfant se retrouve mystérieusement recouvert de perles. Tous cela n'est qu'un prélude aux événements futurs. (p. 14)

Quelle sourdine, quelle percussion plutôt en cet effort de la nomination maternelle!

$\mathrm{Au}$ reste, tout le texte de Louis Cornellier va s'énoncer en séquences phantasmatiques plutôt abracadabrantes: Les affiches font 
une parade de mode pour les voyeurs insomniaques. Les parcomètres, inutiles, songent au suicide. (p. 17) Il va se complaire dans l'évanescente description du vide: Une certaine ambiguité vient couvrir cette chose d'un voile translucide qui embrouille la situation (p. 18): voilà du vide vague! Il va distiller quelques grammes de vitriol (à l'adresse de qui?): Il n'en fallait pas plus pour que des théoriciens de tout acabit viennent tenter d'expliquer ce phénomène illusoire avec leurs thèses ronflantes. (p. 22) Il va se commettre en nombre de naïvetés et de maladresses: Le dos acculé au mur (p. 26) - ils griffonnent des graffitis (sic., p. 59). Voilà donc un premier recueil qu'il eût sans doute fallu, sur le métier... Et à l'épreuve du choix définitif, le jury du prix Octave-Crémazie a eu, somme toute, la main heureuse.

Bonheur de lire, malgré tout, comme s'il suffisait de quelques moments de poésie pour espérer un poète. Ainsi, chez Cornellier, cette image de si évidente beauté: La salive est toujours nue (p. 42); ces saillies de simple profondeur: Le jardin des merveilles peut souvent apparaître dans un espace très restreint (p. 46) et [l]a pensée ne cesse de s'évanouir (p. 47). Juste pour cela qui est juste, qui est poésie, le reste tient de la désolation.

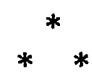

Avec la Terre comme un dessin inachevé ${ }^{8}$, Donald Alarie, en même temps qu'il poursuit assidûment une œuvre de fiction, prolonge le champ poétique qu'il avait amorcé en 1987 avec de très séduisantes proses poétiques intitulées Petits Formats ${ }^{9}$.

Comme l'homme fragmenté du recueil de Louis Cornellier, "L'étrangère", première figure du livre de Donald Alarie, est confinée à une chambre (il faudrait voir ce que ces récentes rêveries de l'intimité, de la chambre comme page et espace mental, doiveńt au beau livre de Louise Dupré, Chambres ${ }^{10}$ ). Cette étrangère, soumise à sa voix intérieure, se tient à une fenêtre (motif qui trouvera son extension technologique dans les analogons du hublot d'avion et du téléviseur), fenêtre de femme où elle s'ádonne à être, à voir - Par la fenêtre de ma chambre, dit-elle, je vois tout (p. 30) -, à voyager: Le front collé contre la vitre de la nuit, elle voyage. (p. 14) Tout l'art de Donald Alarie réside alors dans sa façon d'immiscer au cœur du poème des fils narratifs ténus, des allusions d'événements, des accents de vérité: Il lui arrive de s'attendrir sur sa fiction familiale: [...] Des souvenirs pour les gens qui l'écoutent raconter. (p. 21) Mais toujours le poème, ce souvenir qui vaguement se souvient du monde, continue d'agir comme une mémoire floue du réel; ce qu'il raconte, il le raconte dans la juste dilution impressionniste, dans la rumeur du sous-entendu. 
C'est une mélancolie sur la terre, parmi les paysages, et il ne s'agit plus que de [c]ertains gestes pour confondre le temps et l'espace, pour gruger de l'inédit à même l'ennui (p. 18). C'est l'abandon, le désarroi devant le "caractère approximatif" des "choses": alors, [s]es mains sont disponibles comme des palmes attentives au moindre souffle (p. 34).

Rarement, me semble-t-il, par le recours à de subtils effets d'atmosphères, par la saisie de ces imperceptibles intermittences du corps, du regard et du cœur, par l'énergie de l'immobilité active, n'eston parvenu à rendre si bien une "sensation de pensée", l'indéfinissable qualité d'une "anima». On pourrait encore penser ici au climat durassien, à ces bruissements du monde qui en rendent le silence si oppressant: Un matin de pluie; son cri énorme coupe le monde en deux parties effrayées. Sera-ce la guerre ou le début d'un long silence? (p. 16) Intériorité, certes, écoute du murmure transitoire et de l'instant fragile, scènes fugaces captées en leur fugacité même, mais nulle effusion sentimentale, nulle érosion du sens dans, ce monologue intérieur de "L'étrangère". S'y exprime plutôt, dans le bonheur même de certaines lassitudes, comme une forme moderne du "spleen". Toujours ce texte est servi par un phrasé transparent, une syntaxe rigoureuse et souple à la fois; et je suis de ceux qui aiment bien quand les phrases semblent s'ouvrir pour la première fois et éclatent au centre de la feuille tout à coup lumineuse (p. 20).

Ce désir du poète - tant est grand tout à coup mon désir de voir quelqu'un s'émerveiller (p. 55 ) -, le voilà donc réalisé. Me reste seul, sur la langue, ce malencontreux zeste de tendresse (p. 51)!

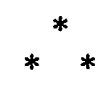

Patiente, en marge des fougues à la mode, procédant par expériences successives, parfois par errements, toujours soucieuse de son authenticité, telle me semble l'œuvre de Denuis Saint-Yves. Je conserve à l'égard de ces entreprises menées dans la "plénière solitude", le respect peut-être distant qu'elles appellent, en même temps que l'espoir tenace qu'elles soient, un jour, mieux mises au jour.

Avec Tranches de ciel ${ }^{11}$, le $9^{e}$ de ses recueils, tous parus, à un rythme posé mais sans relâche aux Écrits des Forges ou au Bien Public, Saint-Yves réitère les raisons - viscérales surtout - de sa venue au poème: si nous allons au poème / c'est que nous avons / de petites bêtes / dans le sang, / de petites bêtes / tourmentées par la lumière (p. 9). À l'instar de tant d'œuvres contemporaines qui actualisent la quête existentielle dans le motif rythmique de l'avancée, ce recueil raconte tour à tour la marche, l'errance nonchalante, la 
course ou la chevauchée dans la tendre indifférence du monde. Ici, l'assentiment à l'univers et à la nature, à la monotonie douce des jours s'effectue sous le mode de ce lyrisme / de l'innocence (p. 46) que le poète a observé chez l'enfant: ainsi le jour / ressemble / au jour' / sans qu'il soit / une fois de plus / question d'y chercher / de vaines réponses (p. 13). Ce courage de l'évidence devant une certaine platitude du réel n'élime pas ici les vertus de l'émerveillement. Saint-Yves parvient souvent, avec une grâce et une simplicité toute pongiennes, à constituer les chosès en tableaux, à faire scène de langage: je regarde le troupeau / en entier dans les verts / pâturages où n'accostent / jamais les navires. / seul le vent / rend compte / d'une voile / à gonfler (p. 18). On comprend mieux alors ce support métaphorique de l'architecture théâtrale (la poutre, la porte de secours, les coulisses, le décor, la scène...) qui vient désigner l'écriture poétique comme un jeu de construction verbale. Le poète, à partir du moment où il a accepté l'état approximatif du monde et malgré sa nostalgie d'une nature "naturelle", malgré son regret de ce pays originel et fondateur (où passent pèlerins, paysans, pionniers et bergers...), n'a plus, par magie d'images, qu'à remettre à son gré la terre en scène, qu'à la compléter comme un dessin inachevé. À l'exemple de ces enfants, chez Donald Alarie: Aussi les enfants qui savent encore tout et qui regardent la terre comme un dessin inachevé auquel il faut absolument ajouter quelques touches personnelles. (p. 81)

Cette saine candeur de l'enfant adonné à jouer (Ne me dérangez pas je suis profondément occupé..., écrivait l'autre, Saint-Denys Garneau...), qui confère à la poésie de Denuis Saint-Yves une sorte de fraîcheur augurale - comme on vient au poème pour les "petites bêtes de lumière" - est parfois distraite par l'épanchement lourdaud de son père: par les soifs / très pures / de ton cœur / pour cristalliser / ta réalité (p. 23). Et l'adulte-enfant ennuie davantage encore quand il porte la voix du "psy" ou celle, tonitruante, du philosophe: je peux bien / faire du critère intellectuel / la base de ma dignité / en ce monde, / et du critère émotif / le sommet / d'une certaine conceptualisation / de ce monde, / mais rien ne me dit / définitivement / ce que sont / le critère intellectuel / et le critère émotif (p. 61).

C'est dire que cette poésie s'accomplit mieux dans la proximité des choses, dans son adhésion spontanée au ciel, aux arbres, aux pierres, bref dans la pensée simple de l'évidence plutôt que dans l'échafaudage du concept nébuleux. Car on trouve, chez Saint-Yves, de belles étrangetés matérielles comme tes seins remontent / la pendule (p. 38) ou encore l'ombre / mais pourquoi / sinon le soleil / qui éclabousse (p. 49); on se plaît à l'évocation éblouie de ces tendres petites choses de la mémoire: maintenant la nuit guette / pour voir si tu allumeras / la lampe. quand tu étais / petit, tous les animaux / 
sauvages connaissaient / ton nom. c'est là, / bien sâr, la plus douce / chose dont tu te souviennes. (p. 89); on s'étonne aussi de telles élucubrations imaginaires: tes lèvres / qui déchaussent / des index tristes (p. 70) ou les parcomètres blêmissent (p. 42). Mais souvenons-nous, chez Louis Cornellier, de cette autre "anthropomorphisation parcométrique»: Les parcomètres, inutiles, songent au suicide. (p. 17) Séquelles surréalistes, bien sûr.

Pourtant il y a toujours, au plus simple chez Saint-Yves, tel ce plaisir de cueillir des simples, ce bel éloge - comme guillevicien et rare devenu - de la tendresse:

La pluie, la nuit, les pleurs.

tout seul on ne peut

que parler de la nuit

sans la nuit, que du

jour sans ses alentours,

que de l'amour sans

le corps.

je pense à ça

avec ta main

dans la mienne. (p. 105)

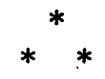

Après les promenades parisiennes et existentielles chez Denise Boucher, après le nomadisme bucolique chez Denuis Saint-Yves et l'effrénée circulation urbaine chez Paul Rousseau, le Piéton immobile suivi de Passiflore ${ }^{12}$, de José Acquelin, pourrait permettre sinon une halte de la lecture, à tout le moins une transition thématique. En effet, la posture oxymorique exhibée par le premier titre de ce recueil incline à rêver une faction active aux mouvements du dehors, une sorte de marche arrêtée par le tourbillon du monde, cela même que suggère la photographie - un mouvement de vitesse en suspens dans une rue - de la page couverture. De plus, d'entrée de jeu, le poète se place sous le signe de la pensée à découvert, de la philosophie didactique: il dit qu'exister c'est être en retard sur l'éternité / c'est vrai ce trottoir n'est pas uniquement trottoir (p. 11). De mémoire, je sollicite ici le très beau vers de Marcelin Pleynet: Le mur du fond est un mur de chaux. Mais à la différence de ce constat étale où s'abolit la perspective des choses, l'oxymoron, qui convoque dans le fracas de deux objets langagiers un objet inédit, tient à un autre ordre du réel, là où dans les mots le monde est capté, accru.

Cette attente d'une collision étincelante, qui crée entre deux mots une tierce vision inouie des choses, sera systématiquement déçue par l'écriture de José Acquelin. Sous prétexte de pensée, la poésie 
s'effondre souvent dans le plus consternant laconisme. Rhétorique ternaire oblige et trois exemples d'"idées " suffisent: un rien m'existe / jusqu'à d'abord (p. 12) - et je crois que nous venons / d'où nous allons (p. 28) - le rien que je suis je le suis bien (p. 30)!

Ajoutons à ces profondeurs, la dérision insipide (la mélancolie est une cigarette / les écureuils sont des nuages [p. 18]) et l'oblitération banale des choses par les choses: le chant d'une cigarette vaut la bouche du stylo (p. 19). Et cela jusqu'à la forme triviale de l'absurde (le ciel est en peau de veau [p. 47]), jusqu'à l'idioterie (sic, p. 50). Ni la naïve détermination figurative (le fer à repasser $d u$ monde [p. 16] et le squelette des infinis [p. 37]), ni d'excessifs effets d'écholalie (un pinson me pince de son silence [p. 17] et les couleurs passent et la pensée se couleuvré [p. 20]) ne parviennent à sortir le lecteur de cette étouffante monochromie, de cet univers sans relief où tout est dans tout.

Pourtant, il suffit d'une sensation heureuse à l'œil : une brise rend les arbres liquides (p. 24) et la pluie n'a jamais vraiment imaginé l'eau triste (p. 90); de la scène d'un jour ordinaire: il y a le soleil les peupliers / un homme en bleu qui donne / des cacahuètes aux pigeons (p. 25); de quelques clichés de charme: la nuit déjeune d'un croissant de lune (p. 53) et les rues sont en réglisse sous la pluie (p. 65); d'un exquis dessin d'enfant: un seul nuage sèche sur la corde à linge / c'est mon enfance (p. 67) et dehors le vent est toujours chez lui (p. 80); d'une douceur subite dans la voix: je sens que tu le sais luce lèvres doúces (p. 63). Alors la poésie paraît. Comment donc, pareilles faveurs de langage peuvent-elles côtoyer telles offenses au silence: le jour me fait gratter sa propre cicatrice à l'écrire (p. 32), la ville se boutonne de réverbères (p. 44) et tes yeux en persiennes zèbrent mon art (p. 58). Serais-je donc "lecteur aveugle"?

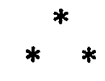

Pour qualifier l'amplitude de souffle du dernier recueil d'André Brochu, Dans les chances de l'air ${ }^{13}$, il faudrait sans doute le placer sous le signe thématique du dynamisme pneumatique, de la diffusion aérienne, de la pulsion respiratoire et vocale, toute rêverie que Bachelard a déjà illustrée dans l'Air et les songes ${ }^{14}$ et qui était aussi présente dans le précédent livre du poète, les Matins nus, le vent $^{15}$. À l'instar de Michael Delisle pour qui la "Chose vocale" se déploie dans l'enivrante matérialité de l'air (Éblouis d'air. / Soulagé d'avoir été ainsi / Sensible au grandiose. [p. 63]), comme Donald Alarie (dont le nom résonne, anagrammatiquement, entre l'air et le rire...) qui vante la qualité sensible de la matière aérienne (L'air se laisse palper. Je marche. Libre.. [p. 56]), André Brochu trouve à son 
tour le lieu de la poésie dans l'élévation feuillue de l'arbre aéré, dans le bruissement des mains s'éployant en la liberté de l'espace. Ainsi dit le beau poème éponyme: Paroles de feuilles haut levées dans le jour / bruissement de mains dans les chances de l'air (p. 133).

L'air est donc ici l'élément favori pour l'inscription du poème, l'immense page de la transparence à traverser, là où l'écriture, calligraphie et crépitement, passe par le visible "transparent" du ciel: Crépitement de bécasses / sur le feu de fougères / l'extrême minute / du ciel passe / refuge / du plus tendre péril / il y a des signes d'air / dans l'air (p. 98). Cette préférence de l'air comme analogon de l'abîme originel et comme élément fondateur de la rêverie matérielle supporte aussi une valeur sensible de genèse; en ce sens, elle trouve son répondant structural dans l'ordonnance du recueil divisé, à l'exemple du temps de la création du monde, en sept parties: "Destins", "Amours ", "Sociétés", "Enfances", "Ironies ", "Natures " et "Larme noire». Le poète, dont émane le Verbe, construit son univers de langage dans la fluctuation du souffle; par le vent, la voix advient. On pourrait entendre en cette prédilection aérienne un goût sous-jacent pour la dispersion volatile ou pour l'effleurement sans appui; chez André Brochu, cette faveur recouvre plutôt une saine, passionnée et généreuse "volubilité", cette "chance" même de "l'air».

Ainsi, le recueil s'ouvre sur le récit d'une naissance - émergence de soi au monde, à son destin, à ses incidences affectives et sociales, tout cela que propose le cheminement de l'œuvre - où la dysphorie compose sans ambages avec le pathos parodique:

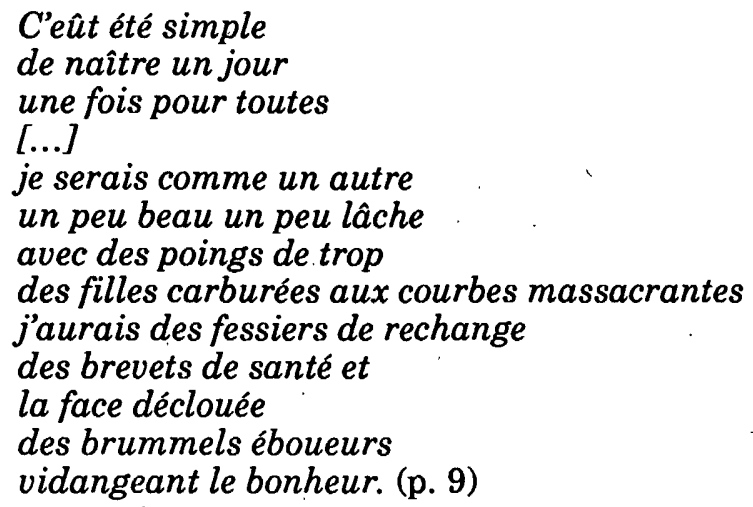

On le voit, rien ici d'évanescent, mais plutôt une sorte de réalisme cru et rieur. Et tout le propos de Brochu, s'il est fondamentalement sollicité par le vertige ou la dissolution spatiale, s'emploie plutôt, d'où la tension poétique qui l'anime, à cultiver les qualités conjointes de l'enracinement et de l'acharnement. 
Ainsi, à la culpabilité d'exister (qui s'exprime en telles images "pieuses": déluge de prières / à la sainteté de / tes jambes nues [p. 35]), le poète oppose la ténacité courageuse d'un "vécrire", [j]'écris avec tout mon courage, activité vitale qui s'exerce dans la conscience heureuse-malheureuse d'être aussi une petite mort: de cette mort et puis / quelle honte me ceint / de ronces non pareilles? je suis la fille / sous le rouge opprobre / qui expire et qui.jouit (p. 13).

Le mal du pays et la honte de la "grande noirceur" (je suis né triste et aigu / comme le bec des pestes / dans le beffroi des âges noirs [p. 17]), sont conjurés par la rage rentrée (d'où le motif insistant du " poing": poing des draps (p. 64), poing de prismes flétris (p. 70), poing des cruautés [p. 76]), par la violence cosmique et comme exacerbée d'un érotisme qui pourrait s'apparenter à celui qui empreint les grands poèmes de Dans le sombre ${ }^{17}$ de Fernand Ouellette. On trouve, chez Brochu, mêmes éclats, même "fulgurance":

ventre mal cuit dans les braises lubriques

ton sexe crache des salives d'astre

à pleine chair dans les pales du vertige (p. 33)

Cette inévitable grandiloquence du discours érotique s'équilibre dans la grâce - un peu mièvre parfois? - du troubadour effarouché devant la gente dame: Je vous salue amie / au doux souris (p. 34); elle s'assouvit dans la tendresse du recueillement: je suis / à jamais retranché de toute / beauté qui n'est pas toi (p. 43); dans les délices capiteuses du roucoulement: puis de revivre toi pleine de grâce / et de souhaits de chrysocale / de péridot et d'astragale (p. 60). Et ce qu'il faut surtout retenir de ces fièvres amoureuses, c'est bien la constante disponibilité du sujet, cet émerveillement devant la différence et l'altérité. En ce sens, si le recueil s'ouvre sur le "je" de la confidence ironique, très tôt il se tourne vers le "tu" qui instaure la fête du monde: tu es un carnaval de désirs / tu aimes tous les corps / le monde est un dimanche (p. 23). Par la rencontre du "je» et du " tu ", il est reconduit au "nous" du collectif, celui pâr lequel le poète s'insurge contre les outrances politiques et toutes les exploitations du pouvoir: les peurs homicides (p. 78), les [f]aces molles (p. 79) de l'histoire et les détournements d'être (p. 81).

On aura maintenant compris que ces "chances de l'air" qui convient la poésie de Brochu s'actualisent à la fois sous le mode d'une utopie et d'une résistance; l'élan de la dilatation aérienne se trouve sans cesse contenu par la solidité d'une incarnation, l'envoûtement filé de la voix en l'air s'accompagne de toute l'énergie d'une diction. Une poétique du "sursaut", ce mouvement qui aérise la pesanteur, est résumée en ce poème cù, sous les mots, "poésie " rime avec «ironie":

Le poème est un roitelet encombré de mots-valises 


\author{
son vol est une entreprise \\ qui fléchit d'une image à l'autre \\ le sursaut seul \\ le tient au-dessus du sol \\ belle méprise
}

roi sans royaume et rire fol (p.139)

La forte cohérence thématique et structurale de ce livre nous fera-t-elle oublier quelques coquetteries stylistiques? Comme le poétisme déterminatif: le hamac des songes (p. 17) et le paon des songes (p. 103); ou telle exubérance allitérative, ce brassage sonore de l'air entre les mots: la faim fricotait son éternél feu de flammes froides (p. 71); ou quelque murmurante trouvaille: Le pluriel de tes regards est une pluie de colibris (p. 49)?

Le plaisir que procure la poésie, croyons-nous - tel celui de son commentaire qui en est, pour le lecteur comme pour le critique, une forme hybride, déviée, maladroitement mimétique sans doute relève de l'adhésion spontanée, de l'empathie viscérale à l'égard d'un autre langage, forme d'exclusive jouissance que viendrait accroître (qui sait?) l'humeur passagère de petites répulsions. En cette matière, tout serait donc affaire de goûts - de ceux qui se discutent, interminablement.

1 Michael Delisle, Chose vocale, Montreal, les Herbes rouges, 1990, 76 p.

2 Jean-Paul Daoust, les Cendres bleues, Trois-Rivières, Écrits des Forges, 1990, $66 \mathrm{p}$.

3 Fernand Ouellette, les Heures, Montréal, l'Hexagone/Champ Vallon, 1987, 118 p.

4 Paul Rousseau, Micro-textes, Trois-Rivières, Écrits des Forges, 1990, 49 p.

5 Denise Boucher, Paris Polaroïd, Montréal, l'Hexagone, 1990, 60 pages.

6 Denis Vanier, les Stars du rodéo, Trois-Rivières, Ecrits des forges, 1990, $74 \mathrm{p}$.

7 Louis Cornellier, Neurones fragmentés, Trois-Rivières, Écrits des Forges; 1990 , $69 \mathrm{p}$.

8 Donald Alarie, la Terre comme un dessin inachevé, Trois-Rivières, Écrits des Forges, $1990,87 \mathrm{p}$.

9 Donald Alarie, Petits Formats, Trois-Rivières, Écrits des Forges, 1987, 61 p.

10 Louise Dupré, Chambres, Montréal, les Éditions du remue-ménage, 1986, $90 \mathrm{p}$.

11 Denuis Saint-Yves, Tranches de ciel, Trois-Rivières, Écrits des Forges, 1990, $107 \mathrm{p}$.

12 José Acquelin, le Piéton immobile suivi de Passiflore, Montréal, l'Hexagone, $1990,93 \mathrm{p}$.

13 André Brochu, Dans les chances de l'air, Montréal, l'Hexagone, 1990, $154 \mathrm{p}$.

14 Gaston Bachelard, l'Air et les songes. Essai sur l'imagination du mouvement, Paris, José Corti, 1965, 306 p.

15 André Brochu, les Matins nus, le vent, Montréal, Editions Trois, 1989, 80 p.

16 Fernand Oucllettc, Dans le sombie dans Poésie, Montréal, l'Hexagone, 1972, p. 139-217. 